\title{
Validation of the fatigue strength assessment of HFMI-treated steel joints under variable amplitude loading
}

\author{
M. Leitner ${ }^{1}$ (D) $\cdot$ M. Stoschka ${ }^{1} \cdot$ Z. Barsoum ${ }^{2} \cdot$ M. Farajian $^{3}$ \\ Received: 6 September 2019 / Accepted: 15 June 2020 / Published online: 23 June 2020 \\ (C) The Author(s) 2020
}

\begin{abstract}
A recommendation for the application and fatigue assessment of the HFMI post-treatment was published by the IIW in 2016. Recently, the therein recommended HFMI design curves in case of constant amplitude loading (CAL) were validated involving test data with different base material yield strengths, increased plate thicknesses, and elevated load ratios. Continuative to this previous work, this paper focuses on the fatigue assessment of HFMI-treated steel joints under variable amplitude loading (VAL). Four test data sets including randomly distributed VAL and a sufficient amount of tested specimens to ensure a statistically verified assessment are investigated. It is shown that an application of the recommended equivalent stress range approach and a further comparison of the test results to the design curves under CAL lead to a conservative fatigue assessment if the recommended value of the specified damage sum of $D=0.5$ is used. Furthermore, an increased value of $D=1.0$ still maintains a conservative design as presented in the study. Based on this work involving the analysed data sets, it can be concluded that the recommended procedure is well applicable and a conservative fatigue design is facilitated.
\end{abstract}

Keywords Fatigue strength · Welded joint $\cdot$ Steel $\cdot$ HFMI treatment $\cdot$ Variable amplitude loading $\cdot$ Equivalent stress range Specified damage sum

\section{Introduction}

The fatigue strength of welded steel joints is generally independent of the base material's yield strength, see IIW recommendation [1]. The application of post-weld-treatment techniques, like the HFMI treatment, is well applicable in order to utilize the lightweight potential of high-strength steel materials [2]. Guidelines for the fatigue assessment [3] under both constant (CAL) [4] and variable amplitude loading (VAL) [5],

Recommended for publication by Commission XIII - Fatigue of Welded Components and Structures

M. Leitner

martin.leitner@unileoben.ac.at

1 Department Product Engineering, Chair of Mechanical Engineering, Montanuniversität Leoben, Leoben, Austria

2 Department of Engineering Mechanics, KTH Royal Institute of Technology, Stockholm, Sweden

3 Fraunhofer Institute for Mechanics of Materials IWM, Freiburg, Germany as well as for quality assurance [6], are developed and published as IIW recommendation for the HFMI treatment [7].

Recently, the applicability of this guideline for the fatigue strength assessment of HFMI-treated steel joints under CAL incorporating increased yield strengths, $R$-ratios, and plate thicknesses is validated by numerous fatigue tests data sets, see $[8,9]$. In case of VAL, Palmgren [10] and Miner [11] proposed a linear damage accumulation, whereas a damage sum $D$ of $D=0.5$ is conservatively recommended in $[1,7]$.

In [12], a study including medium- and high-strength steel joints tested under VAL shows that the real damage sum $D_{\text {real }}$ exhibits a value of $1 / 3<D_{\text {real }}<3$ for most of the analysed data. In case of fluctuating mean stress states, even a lower damage sum of $D=0.2$ is investigated in [13], which is also noted in [1]. In order to assess the fatigue strength under VAL utilizing the recommended fatigue design values in [1], an equivalent stress range $\Delta \sigma_{\text {eq }}$ can be calculated, see Eq. 1.

$\Delta \sigma_{e q}=\sqrt[m]{\frac{1}{D} \cdot \frac{\sum\left(n_{i} \cdot \Delta \sigma_{i}^{m}\right)+\Delta \sigma_{L}{ }^{\left(m-m^{\prime}\right)} \cdot \sum\left(n_{j} \cdot \Delta \sigma_{j}^{m^{\prime}}\right)}{\sum n_{i}+\sum n_{j}}}$

Thereby, $D$ is the specified damage sum, $\Delta \sigma_{i}$ is the stress range and $m$ is the slope above the knee point of the $\mathrm{S} / \mathrm{N}$ - 
Table 1 Overview of fatigue test data sets

\begin{tabular}{|c|c|c|c|c|c|c|}
\hline Data set & Reference & Specimen type & Yield strength (MPa) & Plate thickn. (mm) & $R$-ratio (-) & Load spectrum \\
\hline No. 1 & {$[19]$} & Long. stiffener & S355 & 5 & 0.1 & Straight line \\
\hline No. 2 & {$[20]$} & Long. stiffener & S700 & 8 & -1 & Straight line \\
\hline No. 3 & {$[21]$} & Butt joint & S1100 & 6 & 0.1 & Straight line \\
\hline No. 4 & {$[21]$} & Butt joint & $\mathrm{S} 1100$ & 6 & 0.1 & Gaussian \\
\hline
\end{tabular}

curve, $\Delta \sigma_{j}$ is the stress range and $m^{\prime}$ is the slope below the knee point of the $\mathrm{S} / \mathrm{N}$-curve, $n_{i}$ is the number of load cycles applied at $\Delta \sigma_{i}, n_{j}$ is the number of load cycles applied at $\Delta \sigma_{j}$, and $\Delta \sigma_{L}$ is the stress range at the knee point of the $\mathrm{S} / \mathrm{N}$-curve. For VAL, it is assumed to use $m^{\prime}=2 \cdot m-1$ instead of the value of $m^{\prime}=22$ applicable for CAL $[1,14]$. Previously published studies [15-18] demonstrate that the specified damage sum $D$ for such post-weld-treated steel weld joints varies between 0.2 and 1.0, which maintains a validation of the recommended procedure for a fatigue assessment of HFMI-treated mild- and high-strength steel joints given in [7]. Therefore, this paper focuses on the applicability of the equivalent stress range approach considering specified damage sums in order to validate the fatigue assessment of HFMI-treated steel joints up to a nominal yield strength of $f_{y}=1100 \mathrm{MPa}$ of the base material.

\section{Test data}

In Table 1, the VAL fatigue test data [19-21] used for the validation in this paper is presented. Focus is laid on test data, which includes randomly distributed VAL as well as a sufficient amount of tested specimens to ensure a statistically verified assessment. All cyclic experiments are performed under uni-axial loading at a load ratio of $R=0.1$ (with tensile mean stress) or $R=-1$ (without mean stress). Two different load spectra, namely straight line or Gaussian distribution, are incorporated. Thereby, the straight line spectrum exhibits a shape exponent of $\nu=1$ and the Gaussian spectrum a value of $\nu=2.6$, both with $H_{0}=2 \cdot 10^{5}$ load cycles, see Eq. 2 [22]. Further details to VAL fatigue tests are provided in [23].

$\ln H_{i}=\left[1-\left(\Delta \sigma_{i} / \Delta \sigma_{\max }\right)^{\nu}\right] \cdot \ln H_{0}$

where $H_{i}$ is the cumulative frequency of load cycles for $\Delta \sigma_{i}$, $H_{0}$ is the block size, and $\nu$ is the shape exponent.

Detailed information about the weld specimen geometries, mechanical properties of the base material, HFMI treatment and the VAL testing procedure is given in each reference. In this study, the presented fatigue test data points of each reference are taken as basis for a further evaluation of the equivalent stress range and a final comparison with the recommended fatigue design curve given by the IIW recommendation for the HFMI treatment [7]. Thereby, only the effect of the increased base material strength is considered and no further influences, such as an increased plate thickness or $R$-ratio, need to be taken into account. These further effects are validated in $[8,9]$ for HFMI-treated steel joints under CAL as aforementioned.

\section{Results}

\subsection{Fatigue test results under CAL and VAL}

The fatigue test results of data set no. 1 are shown in Fig. 1 depicted with the maximum nominal stress range. The
Fig. 1 Fatigue test results of data set no. 1 (maximum nominal stress range)

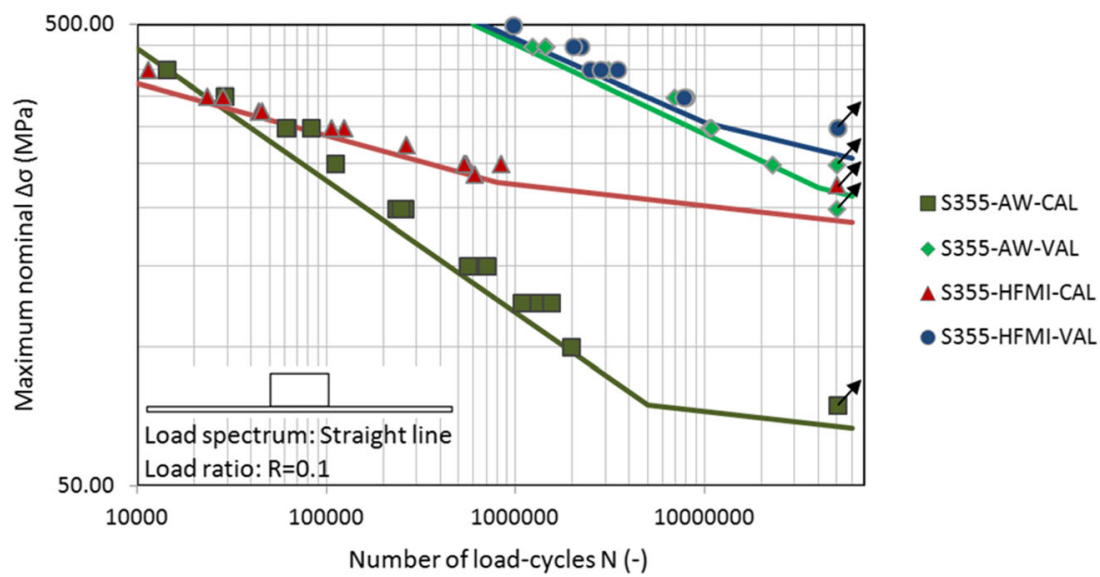


Table 2 S/N-curve parameters of data set no. 1

\begin{tabular}{lcclc}
\hline Test series & $\Delta \sigma(N=2 \mathrm{e} 6)(\mathrm{MPa})$ & $\Delta \sigma(N=5 \mathrm{e} 7)(\mathrm{MPa})$ & Slope $m(-)$ & Scatter 1/To $(-)$ \\
\hline S355-AW-CAL & 97 & 67 & 3.5 & 1.15 \\
S355-AW-VAL & 396 & 217 & 5.2 & 1.09 \\
S355-HFMI-CAL & 204 & 186 & 8.8 & 1.07 \\
S355-HFMI-VAL & 412 & 262 & 5.5 & 1.09 \\
\hline
\end{tabular}

statistical analysis is performed by applying the standardized procedure given in [24] evaluating the $\mathrm{S} / \mathrm{N}$-curve at a survival probability of $97.7 \%$. For the high-cycle fatigue strength with load cycles above the knee point $N_{k}$, a second slope of $m^{\prime}=22$ in case of CAL and of $m^{\prime}=5$ for VAL is applied [1]. Table 2 provides an overview of the statistically evaluated $\mathrm{S} / \mathrm{N}$-curve parameters. As the tests are conducted up to a number of fifty million load cycles, the endurable nominal stress range $\Delta \sigma$ is evaluated also at this level accordingly.

The fatigue test results of data set no. 1 reveal a significant increase of the high-cycle fatigue strength under CAL by a factor of about 2.1 at $N=2 \mathrm{e} 6$ load cycles. In addition, the slope in the finite life regime under CAL increases due to the HFMI treatment, which is in line with the IIW recommendations $[1,7]$. On the contrary, a reduced beneficial effect of the HFMI treatment is observable under VAL. Thereby, a fatigue strength increase of a factor of about 1.04 at $2 \mathrm{e} 6$ load cycles due to the post-treatment is observed; however, at $5 \mathrm{e} 7$ load-cycles, a slightly enhanced benefit of roughly 1.2 is evaluated. As presented in [19] and furthermore investigated in [25], this reduced effect can be majorly drawn to a certain relaxation of the HFMI-induced compressive residual stress state during cyclic loading.

The fatigue test results of data set no. 2 are shown in Fig. 2 depicted with the maximum nominal stress range. Table 3 provides an overview of the statistically evaluated $\mathrm{S} / \mathrm{N}$-curve parameters. As the tests are conducted only within the finite life regime, the endurable nominal stress range $\Delta \sigma$ is evaluated only at a defined number of two million load cycles, which thereby equals the FAT-class. In this case, again a fundamental increase of the fatigue strength due to the HFMI treatment is evaluated under CAL. Thereby, an increase by a factor of about 3.4 in fatigue strength at 2e6 load cycles is observable. However, under VAL, again a reduced benefit by the post-treatment is investigated leading to an increase factor of about 1.3 at $2 \mathrm{e} 6$ load cycles, whereas the tendency is in line with data set no. 1 . The evaluated slopes again fit well to the recommended values.

The fatigue test results of data set no. 3 are shown in Fig. 3 and the results of data set no. 4 are depicted in Fig. 4 with the maximum nominal stress range. Tables 4 and 5 provide an overview of the statistically evaluated $\mathrm{S} / \mathrm{N}$-curve parameters of the two data sets. In detail, under CAL, an increase of a factor of about 1.3 for data set no. 3 and no. 4 is observed at $2 \mathrm{e} 6$ load cycles. Under VAL, this factor equals about 1.1 for data set no. 3 and roughly 1.6 for data set no. 4 again at $2 \mathrm{e} 6$ load cycles. As the run-outs are basically in line with the fatigue test data points in the finite life regime, no knee point is conservatively considered in case of both test series under VAL. In general, the benefit due to HFMI may be reduced for butt joints exhibiting a comparably low stress concentration at the weld toe already leading to a comparably high fatigue strength in the AW condition. In addition, it may be assumed that due to the use of the ultrahigh-strength steel and comparably mildly notched butt joint geometry, no relaxation of the compressive residual stress state as in case of the previous two data sets occurs, which may explain this outcome. Further analysis is scheduled to clarify this behaviour.
Fig. 2 Fatigue test results of data set no. 2 (maximum nominal stress range)

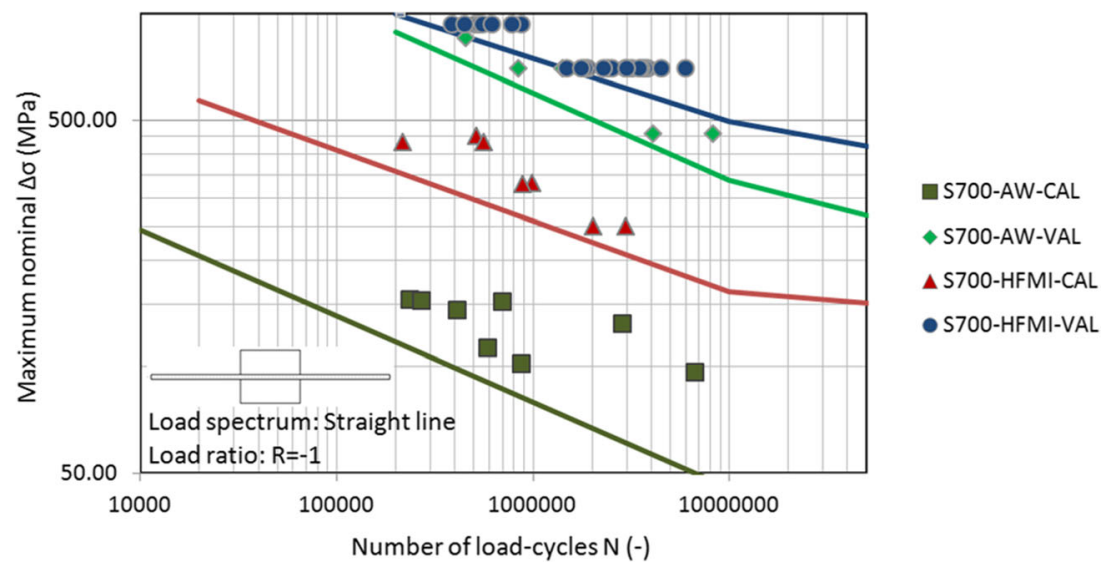


Table 3 S/N-curve parameters of data set no. 2

\begin{tabular}{lcll} 
Test series & $\Delta \sigma(N=2 \mathrm{e} 6)(\mathrm{MPa})$ & Slope $m(-)$ & Scatter 1/To $(-)$ \\
\hline S700-AW-CAL & 67 & 4.1 & 1.76 \\
S700-AW-VAL & 502 & 4.0 & 1.26 \\
S700-HFMI-CAL & 226 & 5.0 & 1.39 \\
S700-HFMI-VAL & 659 & 5.6 & 1.17 \\
\hline
\end{tabular}

\subsection{Fatigue assessment of HFMI-treated joints under VAL}

As introduced, main focus of this work is to validate the applicability of the equivalent stress range approach for HFMItreated steel joints under VAL. Therefore, Eq. 1 is used to calculate the equivalent nominal stress range of the VAL test data for the HFMI-treated joints. For the calculation, the recommended slope $m=5.0$ and $m^{\prime}=9$ is considered. The stress range at the knee point of the $\mathrm{S} / \mathrm{N}$-curve is evaluated according to the applicable design curve based on the IIW recommendations for the HFMI treatment [7]. Herein, it is mentioned that a specified damage sum of $D=0.5$ may be used for the assessment. For comparison purpose, additionally a value of $D=1.0$ is applied within this study. Utilizing these values and the data from each load spectra, the equivalent nominal stress range for each fatigue test data point is evaluated. Finally, the S/N-curve for a survival probability of $97.7 \%$ is statistically evaluated and compared with the applicable design curve for the HFMI-treated joint under CAL.

Figure 5 illustrates the results of the fatigue assessment of data set no. 1 using a damage sum of $D=0.5$. For the 3355 longitudinal stiffener, a design curve of FAT 112 is applicable under CAL according to [7]. Applying the equivalent stress range approach and the value of $D=0.5$, the resulting $\mathrm{S} / \mathrm{N}$ curve is assessed conservatively with a FAT-class of FAT 174 and a slope of 7.2. Therefore, the recommended use of the equivalent stress range approach with $D=0.5$ is validated in this case.

Figure 6 depicts the results of the fatigue assessment of data set no. 1 using an increased damage sum of $D=1.0$. Again, applying the equivalent stress range approach and the value of $D=1.0$, the resulting $\mathrm{S} / \mathrm{N}$-curve is still assessed conservative-
Fig. 3 Fatigue test results of data set no. 3 (maximum nominal stress range)
Fig. 4 Fatigue test results of data set no. 4 (maximum nominal stress range)

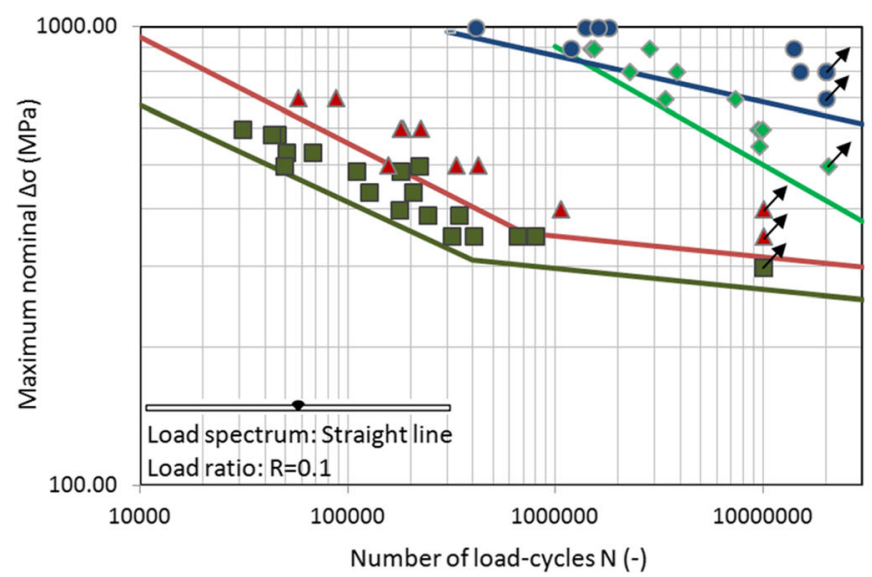

$\square$ S1100-AW-CAL

- S1100-AW-VAL

S S1100-HFMI-CAL

- S1100-HFMI-VAL

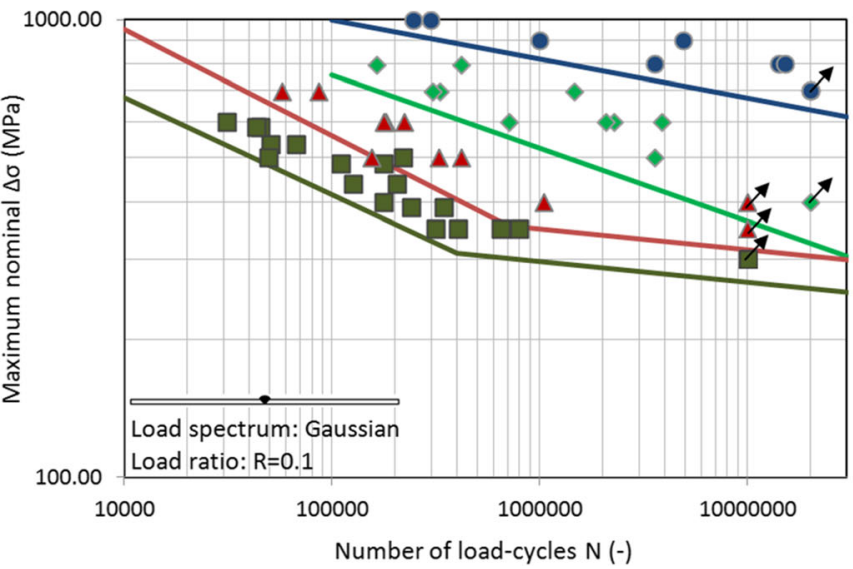

S1100-AW-CAL

- S1100-AW-VAL

$\triangle$ S1100-HFMI-CAL

- S1100-HFMI-VAL 
Table 4 S/N-curve parameters of data set no. 3

\begin{tabular}{llll}
\hline Test series & $\begin{array}{l}\Delta \sigma(N=2 \mathrm{e} 6) \\
(\mathrm{MPa})\end{array}$ & Slope $m(-)$ & Scatter 1/To (-) \\
\hline S1100-AW-CAL & 220 & 4.7 & 1.24 \\
S1100-AW-VAL & 757 & 3.9 & 1.22 \\
S1100-HFMI-CAL & 278 & 4.3 & 1.25 \\
S1100-HFMI-VAL & 808 & 9.9 & 1.25 \\
\hline
\end{tabular}

Table 5 S/N-curve parameters of data set no. 4

\begin{tabular}{llll}
\hline Test series & $\begin{array}{l}\Delta \sigma(N=2 \mathrm{e} 6) \\
(\mathrm{MPa})\end{array}$ & Slope $m(-)$ & Scatter 1/To (-) \\
\hline S1100-AW-CAL & 220 & 4.7 & 1.24 \\
S1100-AW-VAL & 470 & 6.3 & 1.30 \\
S1100-HFMI-CAL & 278 & 4.3 & 1.25 \\
S1100-HFMI-VAL & 774 & 11.6 & 1.18 \\
\hline
\end{tabular}

ly with a FAT-class of FAT 151 and a slope of 7.2. Hence, in this case, also a higher damage sum value of $D=1.0$ may be feasible.

Figure 7 shows the results of the fatigue assessment of data set no. 2 using a damage sum of $D=0.5$. For the $\mathrm{S} 700$ longitudinal stiffener, a design curve of FAT 125 is applicable under CAL according to [7]. Applying the equivalent stress range approach and the value of $D=0.5$, the resulting $\mathrm{S} / \mathrm{N}$ curve is assessed conservatively with a FAT-class of FAT 214 and a slope of 5.6. Therefore, the recommended use of the equivalent stress range approach with $D=0.5$ is again validated in this case.

Figure 8 depicts the results of the fatigue assessment of data set no. 2 using an increased damage sum of $D=1.0$. Again, applying the equivalent stress range approach and the value of $D=1.0$, the resulting $\mathrm{S} / \mathrm{N}$-curve is still assessed conservatively with a FAT-class of FAT 186 and a slope of 5.6. Hence, in this case, also a higher damage sum value of $D=1.0$ may be again feasible.

Figure 9 depicts the results of the fatigue assessment of data set no. 3 using a damage sum of $D=0.5$. For the $S 1100$ butt joint, a design curve of FAT 180 is applicable under CAL according to [7]. Applying the equivalent stress range approach and the value of $D=0.5$, the resulting $\mathrm{S} / \mathrm{N}$-curve is assessed conservatively with a FAT-class of FAT 302 and a slope of 9.9. Therefore, the recommended use of the equivalent stress range approach with $D=0.5$ is again validated in this case.

Figure 10 shows the results of the fatigue assessment of data set no. 3 using an increased damage sum of $D=1.0$. Again, applying the equivalent stress range approach and the value of $D=1.0$, the resulting $\mathrm{S} / \mathrm{N}$-curve is still assessed conservatively with a FAT-class of FAT 263 and a slope of 5.6. Hence, in this case, also a higher damage sum value of $D=1.0$ may be again feasible.

Figure 11 depicts the results of the fatigue assessment of data set no. 4 using a damage sum of $D=0.5$. For the $\mathrm{S} 1100$ butt joint, again a design curve of FAT 180 is applicable under CAL according to [7]. Applying the equivalent stress range approach and the value of $D=0.5$, the resulting $\mathrm{S} / \mathrm{N}$-curve is assessed conservatively with a FAT-class of FAT 414 and a slope of 11.6. Therefore, the recommended use of the equivalent stress range approach with $D=0.5$ is again validated in this case.

Figure 12 shows the results of the fatigue assessment of data set no. 4 using an increased damage sum of $D=1.0$. Again, applying the equivalent stress range approach and the value of $D=1.0$, the resulting $\mathrm{S} / \mathrm{N}$-curve is still assessed conservatively with a FAT-class of FAT 360 and a slope of 5.6. Hence, in this case, also a higher damage sum value of $D=1.0$ may be again feasible.

To sum up, the equivalent stress range approach using a specified damage sum of $D=0.5$ as well as $D=1.0$ leads to a conservative assessment for all analysed data sets. As presented in [19], if not the recommended design curve under CAL is
Fig. 5 Fatigue assessment of data set no. 1 (equivalent nominal stress range). Specified damage sum $D=0.5$

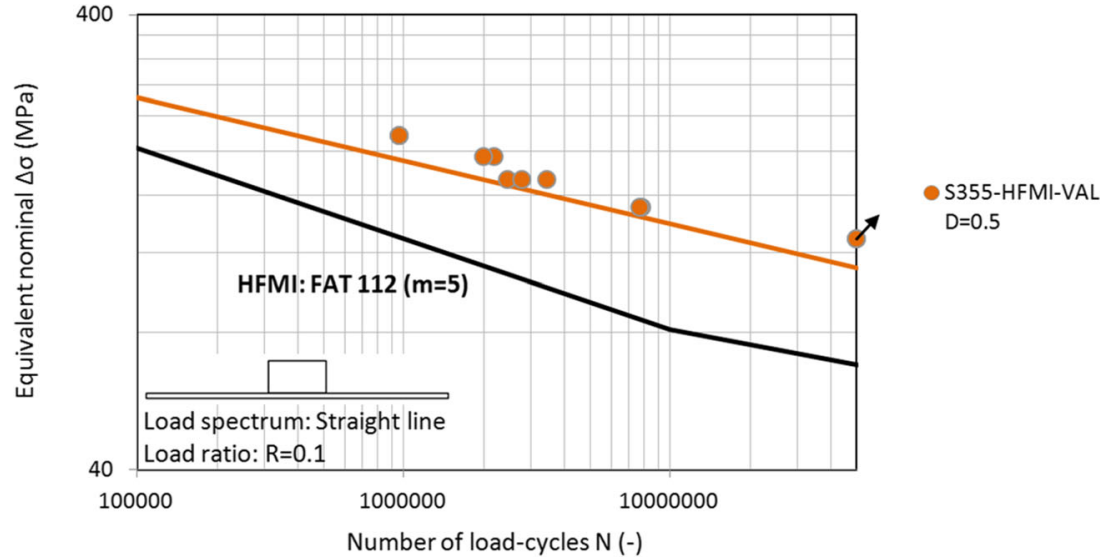


Fig. 6 Fatigue assessment of data set no. 1 (equivalent nominal stress range). Specified damage sum $D=1.0$

Fig. 7 Fatigue assessment of data set no. 2 (equivalent nominal stress range). Specified damage sum $D=0.5$

Fig. 8 Fatigue assessment of data set no. 2 (equivalent nominal stress range). Specified damage sum $D=1.0$

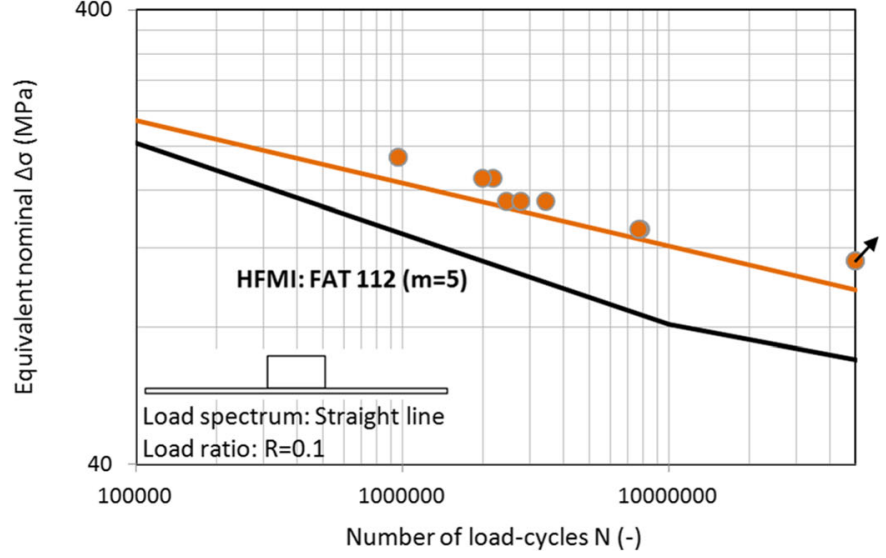

S355-HFMI-VAL $\mathrm{D}=1.0$

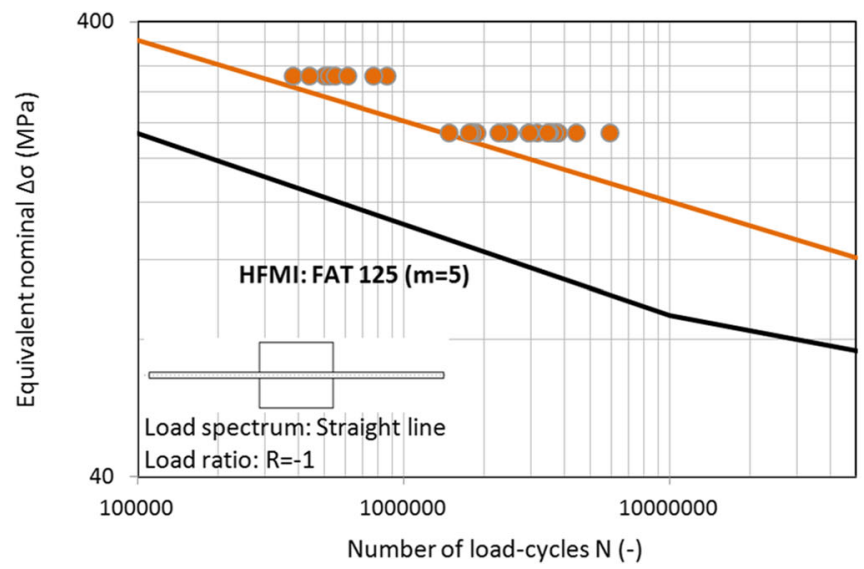

S700-HFMI-VAL $\mathrm{D}=0.5$

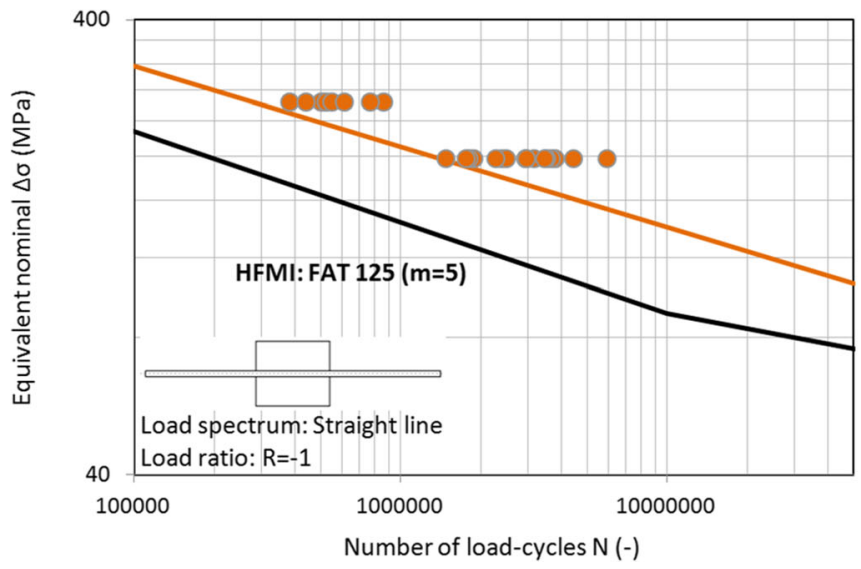

S700-HFMI-VAL $\mathrm{D}=1.0$ 
Fig. 9 Fatigue assessment of data set no. 3 (equivalent nominal stress range). Specified damage sum $D=0.5$

Fig. 10 Fatigue assessment of data set no. 3 (equivalent nominal stress range). Specified damage sum $D=1.0$

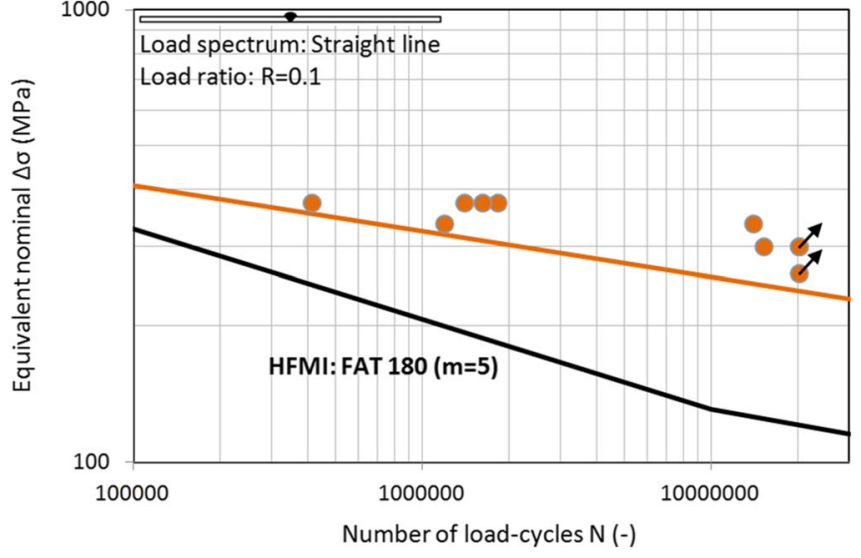

S1100-HFMI-VAL $\mathrm{D}=0.5$

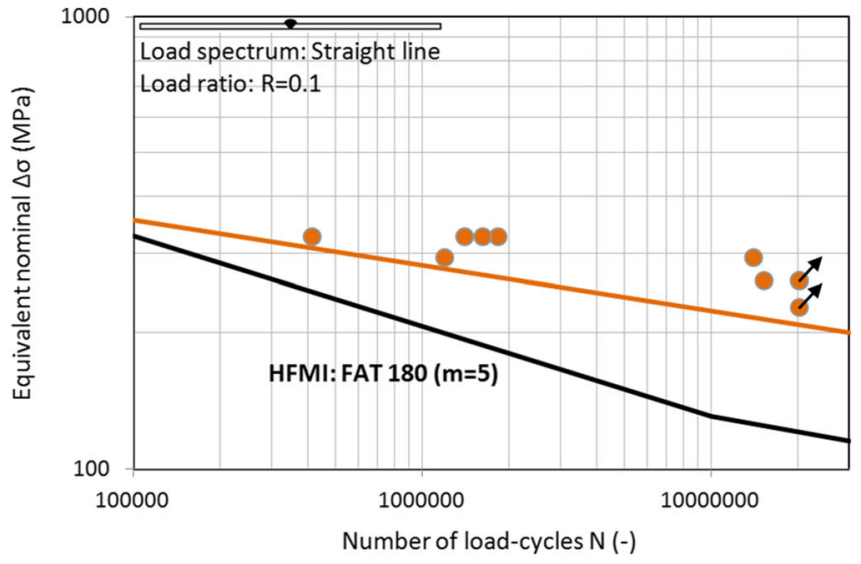

S1100-HFMI-VAL

$D=1.0$

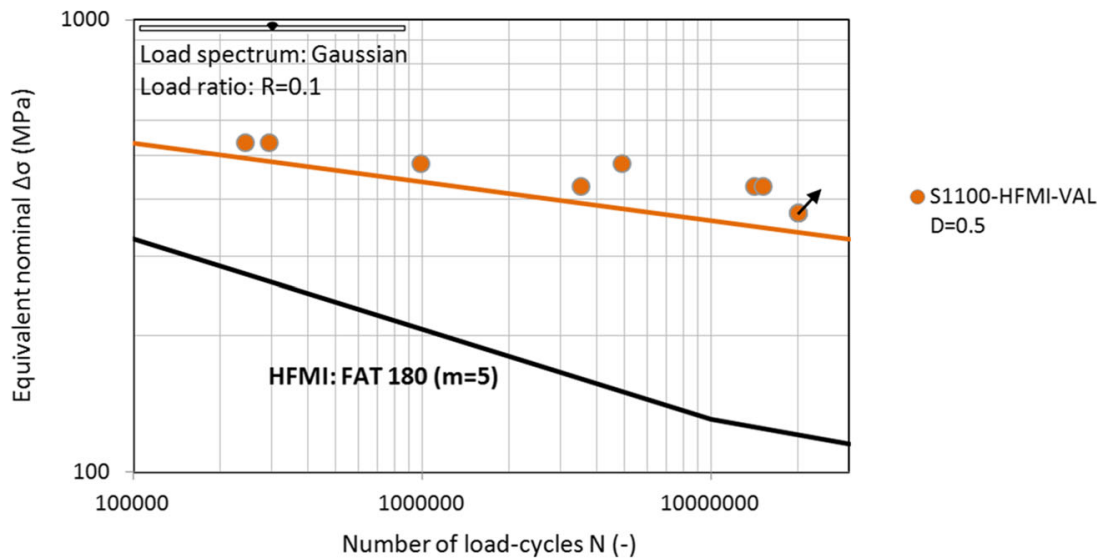

Fig. 11 Fatigue assessment of data set no. 4 (equivalent nominal stress range). Specified damage sum $D=0.5$ 
Fig. 12 Fatigue assessment of data set no. 4 (equivalent nominal stress range). Specified damage sum $D=1.0$

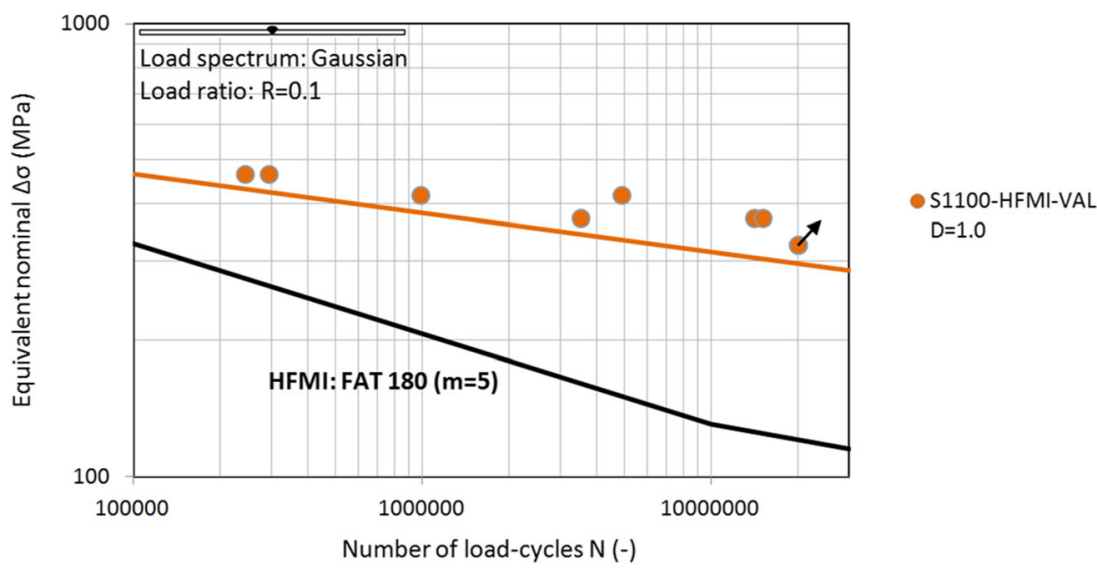

used as basis for the calculation, but an experimentally evaluated $\mathrm{S} / \mathrm{N}$-curve, the damage sum values may be reduced down to $D=0.3$ or even to $D=0.2$ in case of fluctuating mean stress states as also noted in [1].

\section{Conclusions}

This paper aims to validate the applicability of the IIW recommendations for the HFMI treatment in case of HFMItreated steel joints under VAL. Focus is laid on test data, which includes randomly distributed VAL as well as a sufficient amount of tested specimens to ensure a statistically verified assessment, whereas a total number of four test data sets is analysed. Applying the recommended equivalent stress range approach and comparing the results with the design curves under CAL, it is shown that the use of the recommended value of the specified damage sum of $D=0.5$ leads to a conservative fatigue assessment in all cases. Furthermore, an increased value of $D=1.0$ still maintains a conservative design. Based on this work involving the analysed data sets, it can be concluded that the recommended procedure is well applicable and a conservative fatigue design is facilitated. As this study includes only a limited number of currently available data sets, a further validation considering additional $\mathrm{VAL}$ test data of HFMI-treated steel joints is scheduled in the future.

Acknowledgements Open access funding provided by Montanuniversität Leoben.

Open Access This article is licensed under a Creative Commons Attribution 4.0 International License, which permits use, sharing, adaptation, distribution and reproduction in any medium or format, as long as you give appropriate credit to the original author(s) and the source, provide a link to the Creative Commons licence, and indicate if changes were made. The images or other third party material in this article are included in the article's Creative Commons licence, unless indicated otherwise in a credit line to the material. If material is not included in the article's Creative Commons licence and your intended use is not permitted by statutory regulation or exceeds the permitted use, you will need to obtain permission directly from the copyright holder. To view a copy of this licence, visit http://creativecommons.org/licenses/by/4.0/.

\section{References}

1. Hobbacher A (2009) IIW recommendations for fatigue design of welded joints and components. WRC, New York

2. Leitner M, Stoschka M, Eichlseder W (2014) Fatigue enhancement of thin-walled, high-strength steel joints by high-frequency mechanical impact treatment. Weld World 58:29-39

3. Marquis GB, Mikkola E, Yildirim HC, Barsoum Z (2013) Fatigue strength improvement of steel structures by high-frequency mechanical impact: proposed fatigue assessment guidelines. Weld World 57:803-822

4. Yildirim HC, Marquis GB (2012) Overview of fatigue data for high frequency mechanical impact treated welded joints. Weld World 56:82-96

5. Yildirim HC, Marquis GB (2013) A round robin study of highfrequency mechanical impact (HFMI)-treated welded joints subjected to variable amplitude loading. Weld World 57:437-447

6. Marquis GB, Barsoum Z (2014) Fatigue strength improvement of steel structures by high-frequency mechanical impact: proposed procedures and quality assurance guidelines. Weld World 58:19-28

7. Marquis GB, Barsoum Z (2016) IIW Recommendations for the HFMI treatment for improving the fatigue strength of welded joints, Springer

8. Leitner M, Barsoum Z (2019) Effect of increased yield strength, Rratio, and plate thickness on the fatigue resistance of high frequency mechanical impact (HFMI)-treated steel joints, Welding in the World, submitted

9. Ghahremani K, Walbridge S (2011) Fatigue testing and analysis of peened highway bridge welds under in-service variable amplitude loading conditions. Int J Fatigue 33:300-312

10. Palmgren A (1924) Die Lebensdauer von Kugellagern. VDI-Z 58: 339-341 (in German)

11. Miner MA (1945) Cumulative damage in fatigue. J Appl Mech 12: 159-164

12. Sonsino CM, Lagoda T, Demofonti G (2004) Damage accumulation under variable amplitude loading of welded medium- and highstrength steels. Int J Fatigue 26:487-495

13. Zhang YH, Maddox SJ (2009) Investigation of fatigue damage to welded joints under variable amplitude loading spectra. Int $\mathrm{J}$ Fatigue 31:138-152

14. Haibach E (2005) Betriebsfestigkeit, $3^{\text {rd }}$ edition, Springer (in German) 
15. Huo L, Wang D, Zhang Y (2005) Investigation of the fatigue behaviour of the welded joints treated by TIG dressing and ultrasonic peening under variable-amplitude load. Int J Fatigue 27:95-101

16. Tai M, Miki C (2012) Improvement effects of fatigue strength by burr grinding and hammer peening under variable amplitude loading. Weld World 56:109-117

17. Miki C, Tai M (2013) Fatigue strength improvement of out-ofplane welded joints of steel girder under variable amplitude loading. Weld World 57:823-840

18. Mikkola E, Doré M, Marquis GB, Khurshid M (2015) Fatigue assessment of high-frequency mechanical impact (HFMI)-treated welded joints subjected to high mean stresses and spectrum loading. Fatigue Fract Eng Mater Struct 38:1167-1180

19. Leitner M, Ottersböck M, Pußwald S, Remes H (2018) Fatigue strength of welded and high frequency mechanical impact (HFMI) post-treated steel joints under constant and variable amplitude loading. Eng Struct 163:215-223

20. Yildirim HC, Marquis GB, Sonsino CM (2016) Lightweight design with welded high-frequency mechanical impact (HFMI) treated high-strength steel joints from S700 under constant and variable amplitude loadings. Int J Fatigue 91:466-474

21. Pußwald S (2017) Ermüdungsfestigkeitsbewertung ultrahochfester Schweißverbindungen bei variabler Betriebsbeanspruchung. Montanuniversität Leoben, Master thesis (in German)

22. Heuler P, Klätschke H (2005) Generation and use of standardised load spectra and load-time histories. Int J Fatigue 27:974-990

23. Sonsino CM (2007) Fatigue testing under variable amplitude loading. Int J Fatigue 29:1080-1089

24. ASTM International (1998) Standard practice for statistical analysis of linear or linearized stress-life $(\mathrm{S}-\mathrm{N})$ and strain-life $(\varepsilon-\mathrm{N})$ fatigue data, designation: E739-91

25. Leitner M, Khurshid M, Barsoum Z (2017) Stability of high frequency mechanical impact (HFMI) post-treatment induced residual stress states under cyclic loading of welded steel joints. Eng Struct 143:589-602

Publisher's note Springer Nature remains neutral with regard to jurisdictional claims in published maps and institutional affiliations. 